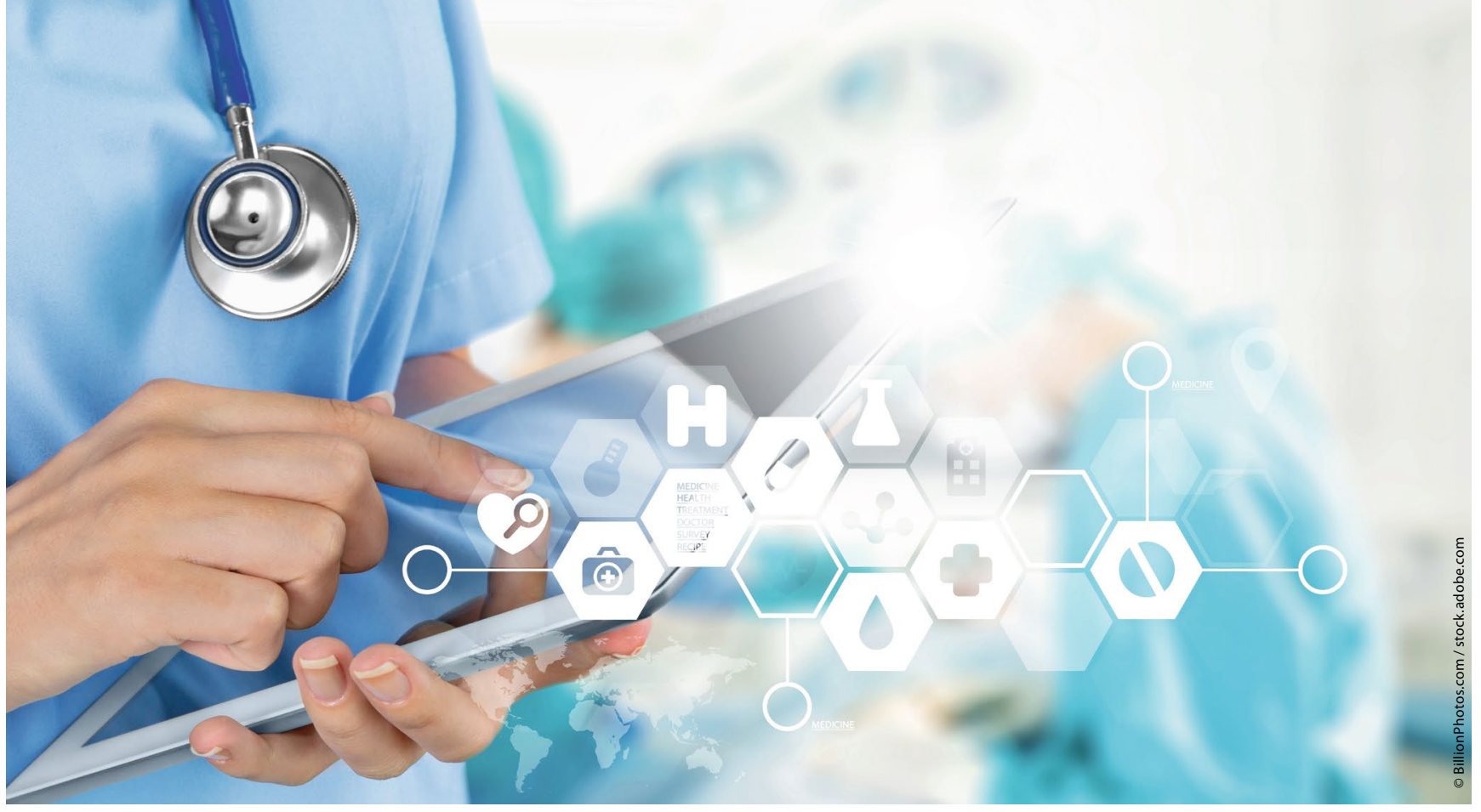

\title{
Die Digitalisierung verändert (auch) die Pflege
}

\author{
Robotik, Sensorik und KI im Berufsalltag Die Pflege befindet sich in einer \\ Krise: Der demografische Wandel und unattraktive Rahmenbedingungen verur- \\ sachen einen zunehmenden Fachkräftemangel. Ein innovativer und moderner \\ Neubeginn zur Gestaltung eines entlastenden Arbeitsumfeldes ist daher unver- \\ meidbar, wenn der Beruf künftig an Attraktivität gewinnen soll. \& Heiko Mania
}

\section{ZUSAMMENFASSUNG}

Digitalisierung in der Pflege bedeutet, ein komplexes Changemanagement mit tiefen Eingriffen in die gewohnten Arbeitsabläufe durchzuführen. Dabei ist mehr der Prozess als die Technik entscheidend. Heutige Pflegedokumentationssysteme können dabei viel mehr leisten als noch vor einigen Jahren: In Verbindung mit Robotik, Sensorik und Künstlicher Intelligenz können nicht nur Pflegemaßnahmen dokumentiert, sondern auch Prädiktionen und damit verbundene Empfehlungen gemacht werden. Die digitalen Möglichkeiten in der Pflege werden folglich das pflegerische Handeln von dem heute meist reaktiven zu einem zunehmend prädiktiven und präventiven Handeln hin verändern.

Schlüsselwörter: Digitalisierung, Robotik, Künstliche Intelligenz, Pflegedokumentationssysteme
D as (Berufs-)Leben wird zunehmend digitalisiert. Daher ist es eigentlich sehr verwunderlich, dass in ca. $70 \%$ der deutschen Gesundheitseinrichtungen die Pflegefachpersonen noch mit Papier und Stift dokumentieren. Diese Dokumentation ist nicht nur sehr zeitaufwendig (bis zu 60\% der täglichen Arbeitszeit), sie wird sehr häufig auch lückenhaft und abrechnungsorientiert durchgeführt. Die aus einer unvollständig geführten Dokumentation resultierenden Gefährdungen für den Pflegebedürftigen sind nicht unerheblich. Und wurde bisher der finanzielle Aspekt als Grund der schleppenden Digitalisierung in der Pflege aufgezeigt, bietet beispielsweise das Krankenhaus-Zukunftsgesetz (KHZG) hier eine große Chance, echte Entlastung der Pflegenden durch die Digitalisierung zu erreichen. Voraussetzung für den Erfolg solcher unterstützenden Technologien ist es jedoch, die damit zusammenhängenden Aktivitäten nicht als IT-Projekte anzugehen - wie es so häufig in der Vergangenheit üblich war. Digitalisierung in der Pflege bedeutet eben nicht nur Software, Computer oder Roboter einzusetzen, sondern umfasst ein komplexes Changemanagement mit tiefen Eingriffen in die gewohnten Arbeitsabläufe der 
Fachleute. Bisher analoge Inhalte oder Prozesse der Pflege werden in eine digitale Form und Arbeitsweise umgewandelt und miteinander vernetzt. In der digitalen Transformation ist der Prozess die Lösung - und nicht die Technologie. Daher ist dieses Prozesswissen unentbehrlich und eine Teilhabe von Pflegefachpersonen hier unbedingt notwendig.

\section{Die Qual der Wahl: Pflegedokumentationssysteme}

Eine weitere wichtige Rolle für die digitale Transformation spielt außerdem die Wahl für eines der digitalen Pflegedokumentationssysteme. Diese haben sich in den letzten Jahrzehnten mit den zunehmenden technischen Möglichkeiten noch einmal deutlich weiterentwickelt. Dabei ist aktuell dennoch der größte Wetterbewerber der Stift und das Papier, so wie einst von Florence Nightingale begonnen. Diese Art der Dokumentation hat jedoch immer einen fixen Umfang, die Formulare bleiben ja stets gleich groß (egal wie pflegeaufwändig der Patient ist) und Kennzahlen zur Steuerung der Maßnahmen und Qualität sind nur aufwendig zu gewinnen.

Mit der Jahrtausendwende waren dann die ersten Pflegeinformationssysteme (PfIS) verfügbar. Meist waren sie Teil der monolithischen Krankenhausinformationssysteme (KIS) oder Heiminformationssysteme (HIS). Hier wurden jedoch häufig aus den Papierformularen digitale Abbilder geschaffen und damit leider nicht selten auch Datensilos. Denn die Ergebnisse solcher Formulare hatten meist keinen weiteren Einfluss auf andere digitale Prozesse. Zudem waren diese Systeme in der Regel auf eine administrative und abrechnungs-relevante Dokumentation fokussiert und daher häufig nicht mobil am Point-of-Care (am Patienten oder Bewohner) einsetzbar. In der Folge musste sich der Prozess häufig an den technischen Möglichkeiten anpassen und Pflegefachpersonen beispielsweise an den festen Computern am Pflegestützpunkt dokumentieren, obwohl die Daten eigentlich am Bett gewonnen und benötigt wurden. Vor ungefähr zehn Jahren traten dann die ersten spezia- lisierten, digitalen Pflegedokumentationssysteme in Erscheinung. Diese sollten die Pflegefachpersonen mit integrierten Pflegefachsprachen, -klassifikationen und ersten Teilautomatisierungen bei einem leichter geplanten und dokumentierten Pflegeprozess in hoher fachlicher Qualität unterstützen. Durch dessen Nutzung wurden zudem erste Prozesskennzahlen generiert, die auch zur Qualitätsbewertung, Versorgungs- und Personalsteuerung herangezogen werden konnten.

\section{Automatisierung, Künstliche Intelligenz und Sensoren verändern die Pflege}

Die modernsten, pflegerischen Dokumentationssysteme sind die Pflege-Expertensysteme (PES). Diese digitalen Plattformen lassen sich als Software verstehen, die Pflegefachleute bei der Lösung ihrer komplexen Aufgaben wie ein Pflegeexperte unterstützen kann, indem sie Daten der Pflegenden und von Sensoren analysiert und Handlungsempfehlungen aus einer Wissensbasis mittels Künstlicher Intelligenz (KI) ableitet. Diese Systeme sind so entwickelt, dass sie über moderne, digitale Austauschstandards (Interoperabilität) umfangreiche Daten mit anderen Softwaresystemen im Gesundheitswesen austauschen können und so die fehleranfälligen Mehrfacheingaben von Daten nicht mehr notwendig sind. Die meisten Schritte des Pflegedokumentationsprozesses sind in einem PES automatisiert, so dass ein großer Teil des Dokumentationsaufwandes reduziert und signifikant Zeit gespart wird. Zudem können diese „digitalen Pflegeexperten“ auch pflege-sensitive Risiken und zunehmend auch Pflegephänomene auf Basis der unterschiedlichen Datenquellen vorhersagen (Prädiktion). Das bedeutet nicht nur, dass ein PES die Dokumentation schneller erledigt, sondern dass durch dessen Vorhersage von Risiken und Phänomenen auch viel schneller präventive Pflegeinterventionen vorgeschlagen und von den Pflegefachleuten entsprechend umgesetzt werden können (Abb. 1). So kann ein Pflege-Expertensystem beispielsweise nicht

Abb. 1: Die digitale Transformation in der Pflegedokumentation

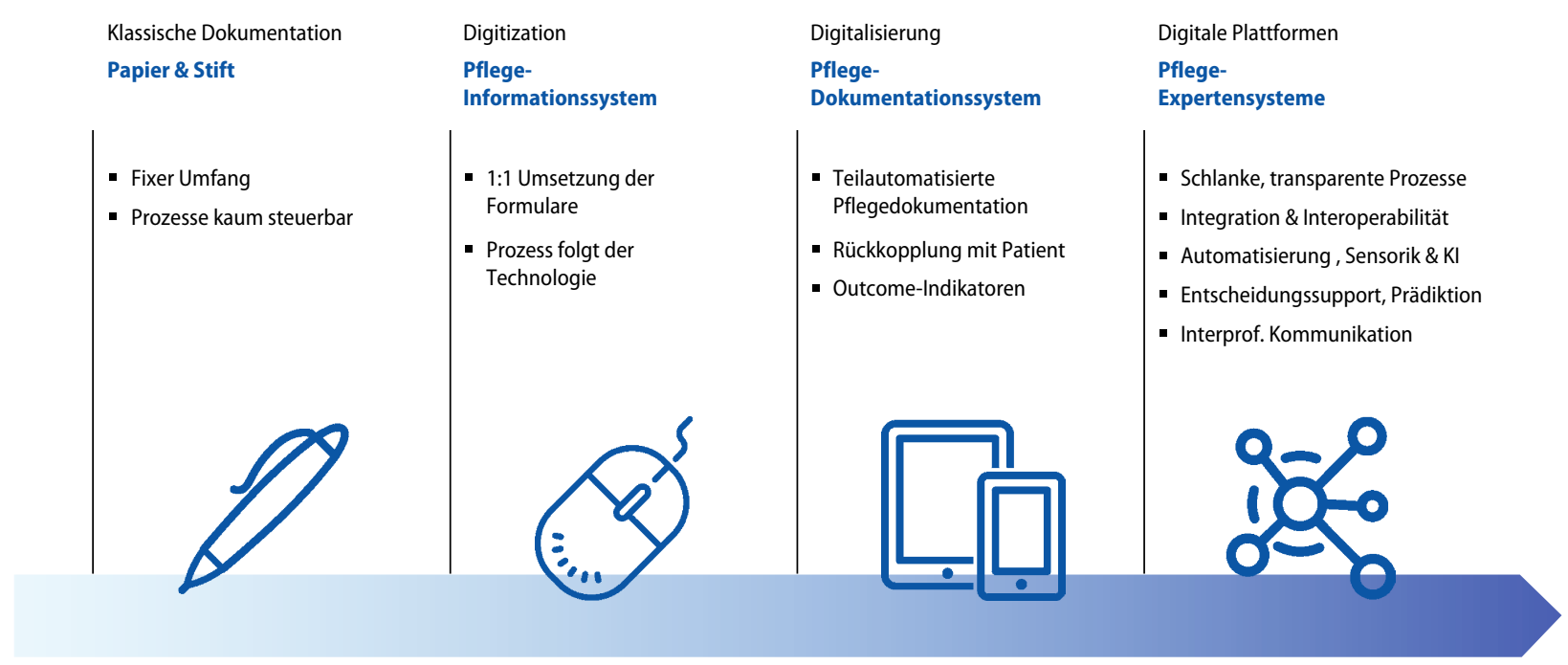

Digitization = Umsetzung von anlaog zu digital DigitALISIERUNG = Digitization. + Geschäftsmodell 
Abb. 2: Pflege-Expertensoftware mit zusätzlichen digitalen Services

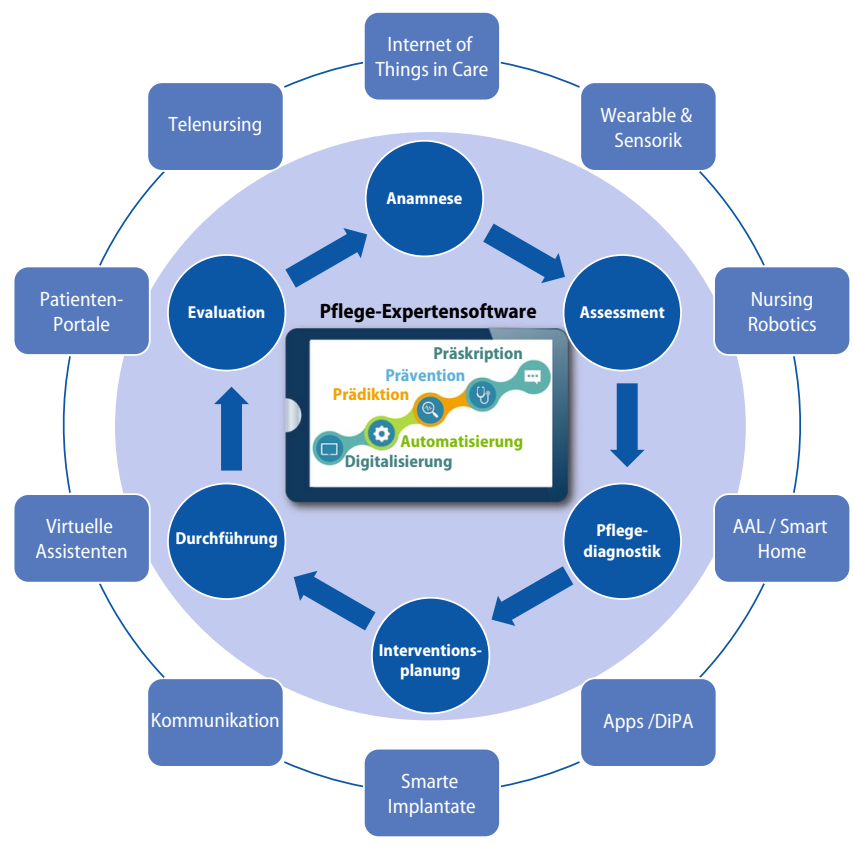

nur das Dekubitusrisiko eines Patienten erkennen, sondern auch direkt auch präventive Maßnahmen vorschlagen oder gar Antidekubitus-Hilfsmittel im Kontext selbst anfordern. Aber auch smarte Pflegehilfsmittel spielen eine zunehmende Rolle im pflegerischen Arbeitsumfeld. So kann der intelligente Trinkbecher nicht nur erfassen wie viel sein Nutzer getrunken hat, er erstellt automatisch auch das Trinkprotokoll oder kann seinen Nutzer an das Trinken selbst erinnern. Ein weiteres Beispiel ist der „Blasen-Füllstandsensor". Dabei handelt es sich um einen kleinen, mobilen Ultraschallsensor, der auf die Bauchdecke des Nutzers fixiert wird. Dieser misst nun kontinuierlich den Füllstand der Harnblase und berechnet durch Künstliche Intelligenz, wann der Patient die nächste Harnblasen-Entleerung erfahren wird - bevor es zu einem möglichen „Malheur“ kommt.

Diese neuen Möglichkeiten mit Hilfe von Sensorik und Künstlicher Intelligenz fachliche Vorhersagen zu treffen, verändert zugleich die Art und Weise pflegefachlichen Handelns. So können unerwünschte Ereignisse beim Patienten und damit einhergehende Pflegehandlungen vorzeitig vermieden werden. Dies wiederum spart Zeit und Aufwand für die Pflegenden und erhöht die Versorgungsqualität für den Pflegebedürftigen. Die Patientensicherheit und Reduktion von Risiken erhalten somit eine ganz neue Handlungsebene (Abb. 2).

Die Anbieter von heutigen Pflege-Expertensystemen arbeiten aber schon an weiteren, entlastenden Funktionalitäten. Mittels der sogenannten „Präskription“ wird beispielsweise aus allen vorhandenen Pflegedaten für einen Patienten in seinem individuellen Setting der beste Pflegepfad vorgeschlagen. Dafür vergleicht das System alle ähnlichen Versorgungsdokumentationen und -settings mit den entsprechenden Pflegeergebnissen und leitet davon den quali- tativsten Versorgungsplan ab. Die Pflegefachpersonen und Patienten können somit von den eigenen (pflegerischen) Daten profitieren.

\section{Zukunftsmusik oder konkrete Pläne?}

Die Möglichkeiten der digitalen Transformation werden das pflegerische Handeln von dem heute meist reaktiven zu einem zunehmend prädiktiven und präventiven Handeln hin verändern. Auch wenn es sich noch wie Science-Fiction anhört, so sind die Technologien bereits vorhanden. Durch das Krankenhauszukunftsgesetz wird die digitale Pflege- und Behandlungsdokumentation nicht nur gefördert, sondern auch bis zum 1.1.2025 explizit gefordert. Andernfalls drohen hier den Kliniken empfindliche Abschläge. Die Pflege-Expertensysteme mit ihren Funktionen zur Unterstützung der Pflegeprozessdokumentation passen genau in diese Förderung und werden eine deutliche Verbreitung in Deutschland erleben. Sie bilden eine pflegerische Basis-Plattform, auf der dann unterschiedlichste digitale Pflege-Services aufsetzen können.

>II

\section{(1) FAZIT}

Bei der Einführung und Umsetzung digitaler Projekte ist auf ein angepasstes, iteratives Vorgehen zu achten.

Die Pflegefachpersonen sind gut vorbereitet in die digitale Arbeitswelt mitzunehmen und zu beteiligen. Der Nutzen und die Entlastungseffekte stellen sich dann meist schnell und spürbar ein.

Im Zuge der Digitalisierung wird sich der Beruf der professionell Pflegenden sehr verändern: Prädiktion und Präskription werden die Pflegemaßnahmen zukünftig beeinflussen.

\section{BUCHTIPP
V. Kubek, S. Velten, F. Eierdanz,
A. Blaudszun-Lahm (Hrsg.) Digitalisierung in der Pflege}

Springer Verlag 2020

ISBN 978-3-662-61372-6, 39,99€ (eBook)

ISBN 978-3-662-61371-9, 48,59€ (Softcover)

\section{Autorenkontakt:}

Heiko Mania, M.Sc., MBA ist Krankenpfleger und Geschäftsführer der NursIT Institute $\mathrm{GmbH}$ in Berlin

E-Mail: mania@nursit.intitute 